\title{
166416 JanvierRelation du Médecin Torrini de la maladie, et des causes de la mort de la Duchesse de Savoye Françoise de Valois
}

\section{Alessandro Bargoni}

\section{OpenEdition}

Journals

\section{Edizione digitale}

URL: http://journals.openedition.org/studifrancesi/35597

DOI: 10.4000/studifrancesi.35597

ISSN: 2421-5856

\section{Editore}

Rosenberg \& Sellier

\section{Edizione cartacea}

Data di pubblicazione: 1 juillet 2005

Paginazione: 106-114

ISSN: 0039-2944

\section{Notizia bibliografica digitale}

Alessandro Bargoni, «1664 16 JanvierRelation du Médecin Torrini de la maladie, et des causes de la mort de la Duchesse de Savoye Françoise de Valois», Studi Francesi [Online], 145 (XLIX | I) | 2005, online dal 30 novembre 2015, consultato il 19 avril 2021. URL: http://journals.openedition.org/ studifrancesi/35597 ; DOI: https://doi.org/10.4000/studifrancesi.35597

\section{(c)}

Studi Francesi è distribuita con Licenza Creative Commons Attribuzione - Non commerciale - Non opere derivate 4.0 Internazionale. 


\section{Relation du Médecin Torrini de la maladie, et des causes de la mort de la Duchesse de Savoye Françoise de Valois ${ }^{49}$}

Immanem severitatem publici stati acerbius ne deplorem an mei, cuius est saeni doloris causas funestissima enarratione disserere. Heret faucibus vox ac inter lugubres nenias, heus quam prestaret muta persolvere parentalia ingentibus enim oppressa curis stupet mens, nictant occuli, calamus nutat, et pupillae in lachrimas dissolutae funestos eluere characteres inutili conatu procurant. Aggredior tamen quia dolori nimio facit imperium, et iuvat Regy occasus acerbitatem ineluctabilis, ac prefinitae necessitatis recordatione lenire. Describam itaque et graphice pro ut medicum decet, et brevi pro ut mœrentem.

Regia Princeps Francesca Valesia Sabaudiae Ducissa Cypri Regina à decem men-

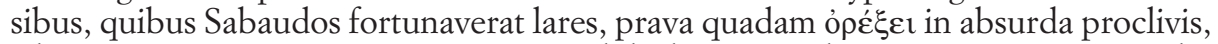

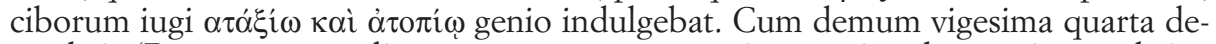
cembris (Postquam per aliquot praegressas noctes in somnis calere acriter, sed sine febre comperta foret) manifeste febricitavit: Iterum[que] $26^{a}$ et $28^{a}$, periodo nempe tertianaria. Vel non novit, vel sprevit ipsa dolores suos, quo tempore ob supremam valetudinem et supremum funus (Heus quam lugubre) Regiae Matris supremis itidem et ipsa torquebatur angoribus. Hinc causarum cumulus ingens. Pertinaciae vigiliae, inediae moerores, atq[ue] in molli aetate mollique sexu terrores tantum non panici. Post quae tandem mentis vigesima octava perennis corripuit ignis, et febris continens, cuius proinde initium ab ea die fas est exordiri.

Ad $4^{\text {am }}$ usque continens febris, et quae synocham parachimastriam simularet. $4^{\mathrm{am}}$ diarrhoea, deiectiones biliosae, variae. Quinta fluxus $\kappa \alpha \tau \mu \eta v i ́ \omega v$ intempestivus, et ganeus, febris remittitur. Sexta febris remissior. Septima $\chi \alpha \tau \alpha \alpha \mu \eta \eta_{\nu} \alpha$ cessant,

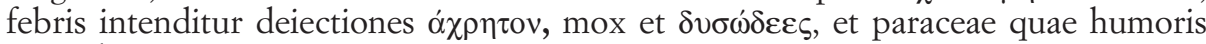
cacoethe $\backslash r /$ iam testarentur.

Exacerbatis circa vesperam per totum morbi processum quotidianis accessionibus perseveratura ${ }^{50}$, cum quadam sed levi circa meridiem anadyplosi. Nona lumbricus vivens, subruber, per alvum ductus, deiectiones frequentiores et variae. Decima lienteria. Undecima exacerbata omnia 'A $\lambda \iota \sigma \mu o ́ \varsigma$, sitis inexausta, gravis et frequens anhelitus, tussis molesta, genarum rubor, calor ó $\xi u ́ s$ in digitorum palpis, suspicis epigenesis in pulmones, et peripneumoniae. Interea subsultus in carpo, et motus iectigativi / $\left(\mathrm{f}^{\circ} 2 \mathrm{r}\right.$ ) vel acrimonia sua continentes dia $\backslash \mathrm{r} / \mathrm{rhaeas}$ provocaturus, vel malitia

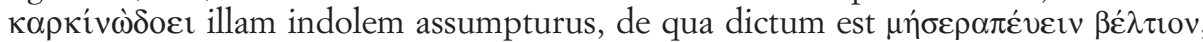
vel denique incremento intestini aditum obstructurus cuius emphrati remoratae alvinae sordes in ileos ${ }^{51}$ dysenterias, cruciatus, et feras anadromes pullularent.

Thorax angustum intra cuius parietes coarctati pulmones inspirationis remora[m] quandam experirentur, unde conclusis, nec diflatis halitibus et geminata pro angustiae compensatione antistrophe, nil nisi incandescere superesset. Pulmonum substantia fusca nigredine torrefacta, arida et exsucca, livida, lurida sine sistrophe, sine tuberculo, nisi quatenus ex altero lobo expressus pauculus liquor saniei praetulit speciem, ex altero expraessus cruor speciem attramenti. Verbo tota substantia tantum non sphacelata. 
Haec est area in qua veluti in luto natura haesit, et subsidiarijs in viribus distituta imperio cessisse suo reapse convincitur. Succensa nempè penitissimis intrò visceribus flammâ primum quidem ebullire biliosi atrique succi, quorum ingens in corpore apparatus, mox rapi in proxima et naturalia viscera eorumq[ue] tonum et aeconomiam perturbare. Illic acrimonia sua solicitant diarhaeam et festinato ante[m] concoctionem $^{52}$ expultricis irritamento lienteriam. Illic et hepati inurunt phlogosim et semina iaciunt bilis ultro regenerandae. Mox altius progressa ebullitione fermentantur, pu-

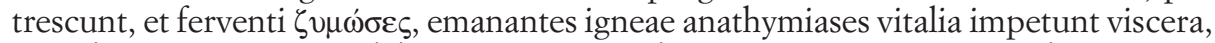
ut trahant in consensum labis. Primi ipsi in discrasiam proni sensere pulmones, qui

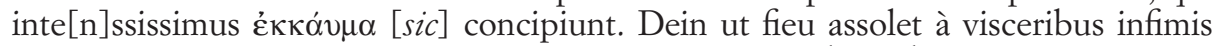

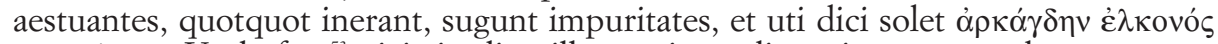

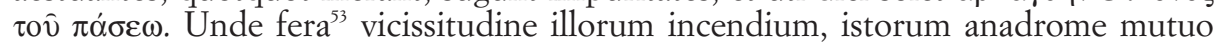
foveantur et foveant. Verum quoniam aquea magis illuvies devolvebatur in alvum par erat ferri ad pulmones paucam humiditatem, quae intenssissimo estu ${ }^{54}$ protinus soluta in auras systrophis aut tuberculi nulla[m] occasionem relinqueret. Solae itaque aereae et igneae illuc appulere impuritates eò deteriori Fato ${ }^{55}$, quo quia siccitas lima

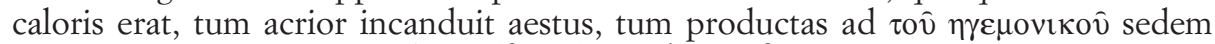
meteorsmus iectigassiones,${ }^{56}$ et $/\left(f^{\circ} 1 \mathrm{v}\right)$ prodromi futurae $\pi \mu v \phi v s i r u s$. Decima $4^{a}$. Paraphrenitis $\backslash 16^{\mathrm{a}} \mathrm{coma} /$, neq[ue] tantum praestitere ex artis praescripto usurpata praesidia, quae poulmonum phlogosim, humoris fervorem acrimoniam, cacoetheiam corrigerent. Phlebosomiae, blanda cathartis, refrigerantia, humectantia, renutrientia,

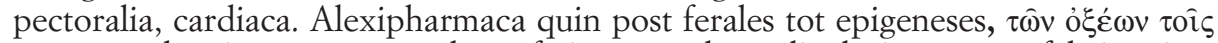
ó $\xi \varepsilon \sigma$, tandem increscente morbo et fatiscente robore die decima octava febris prima ipsa accessionis hora fato concederet.

Lubuit licuitq[ue] secto Regio cadavere ea perscrutari, quae assertam pridem

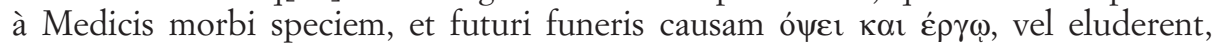
vel comprobarent; praecipuè vero num pulmonum aestum ac phlogosim par esset arguere. Ac protinus, en prima fronte oblatae exsuccae carnes, resiccati musculi, ac rore exhausti suo ingentis argumentum incendij, quod tum aqueam, tum pinguem humiditatem dissipavit. Contentae Partes peculiari adèo sym $\backslash \mathrm{m} /$ etria distinctae, ut sinistrae dexteris essent respective grandiores, cum interim cava ultra naturalem proportionem amplitudinis superaret aortam, quod quidem posita periodo sanguinis mirum quantam ad internas systrophas facere debuerit propensionem.

Abdominis cuncta viscera emuncta item et arida, quaeq[ue], nec exprimenti udorem concederent. Nempè non tam vacuatis pertinaci fluxu, quam et discussis et in fuligines elevatis humoribus à fervore acerrimo, à quo et ventriculus, et intestina quandam rubedinem contraxere, veluti et hepar exanguem habitum nec non $\kappa \lambda \omega p o ́ v$, ac pallido flavam tincturam. Vacua sua bile cystis, quia partim in alvum cesserat pro $\dot{\varepsilon} \rho \varepsilon \theta \imath \mu \omega$ diarrhaeae partim insigni ebullitione meteorismum conceperat. Intestinis hoc unum non levis momenti inerat: tumor divulsus, asper carnei quantum locus pati patest coloris, alicubi etiam sub viridis, schynosus, òvé $\lambda \kappa \omega \tau$ tos, et verbo tumor carcinomatodes, qui partem ceci et coli totum initium etiam infra valvulam intro succingebat. Soboles ista quidem tum melancholici succi, tum sordium quae in ceci bursa plus aequo grandiori collectae ab aliquot mensibus potuerunt in eius, et coli substantia systrophem parere, et ultimis hisce diebus titillamento suo alvum ${ }^{57}$ iugiter proritare: sed in qua tamen proclive est, syderis nostri fixam perniciem serio

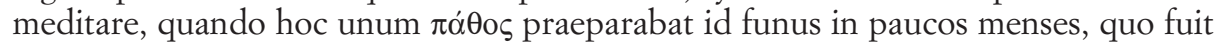

(52) fera sur $+?+$

(53) ist $u$ sur ista.

(54) Le $-f$ initial a été corrige en majuscule.
(55) + atq[ue]+

(56) alvum sur album.

(57) + cong+ 
alterius festinatione praeoccupatu[m]. Quid ni enim sobolesceret tumor, ac servatae nunc vitae, tunc falcem summitteret $/\left(f^{\circ} 2 \mathrm{v}\right)$ deliria usque promovit, tum denique pabulum adintrà querens exaestuans flamma radicalem ipsum humorem adeò depasci debuit, ut dissoluto funditus partis tono quandam veluti syderationem \inferret/ ac

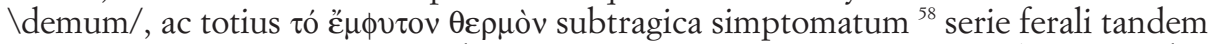
cum scena corrueret. Ergo et calamus corruat ac aeterno ${ }^{59}$ moero[r] em ${ }^{60} \backslash$ in tumulo/ provolutus $^{61}$ cum Principe vita fatiscat. Taurini 16. Januarij 1664.

Bartholomeus Torrini.

\section{Considerazioni medico-cliniche}

Il documento inedito rinvenuto presso l'archivio di Stato di Torino presenta aspetti di indubbio interesse sotto molteplici punti di vista. Quello storiografico generale in primo luogo, ma anche quello dello studio della Storia della Medicina e dell'arricchimento dei profili biografici dei personaggi attori della vicenda.

La "Relation du Médecin Torrini de la maladie, et des causes de la mort de la Duchesse de Savoye Françoise de Valois" può concettualmente essere suddivisa in quattro parti. L'introduzione di stile letterario apre con la dichiarazione di Bartolomeo Torrini del suo dolore per la scomparsa della duchessa a pochi giorni di distanza della Reggente Cristina di Savoia. Successivamente si passa alla descrizione dettagliata del decorso clinico. È poi la volta della descrizione dell'autopsia del cadavere della Principessa. La chiusura del documento è affidata a considerazioni filosofiche sul triste destino delle due duchesse di Savoia e termina con il congedo dalle spoglie della principessa che vengono affidate alla tomba.

Il linguaggio utilizzato nella estensione di questa relazione con continui richiami alla dottrina del Corpus Galenico-Ippocratico, legano fortemente Bartolomeo Torrini alle visioni strettamente accademiche del suo tempo. Per contro la narrazione del susseguirsi degli eventi patologici, dei sintomi e dei segni clinici, è attentamente condotta ed esposta secondo la corretta cronologia dei fatti, frequentemente intercalata e chiosata da notazioni e interpretazioni dottrinali secondo i canoni degli autori antichi. Colpisce quindi l'atteggiamento dicotomico tra il descrivere in modo chiaro e comprensibile fenomeni oggettivi rilevati con l'uso dei sensi e contemporaneamente fornire la loro spiegazione in chiave teoretica abbandonando il ragionamento piano e razionale. Un esempio di questo atteggiamento scientifico si osserva nel testo della relazione quando Bartolomeo Torrini, nel corso dell'esame del cadavere, rileva un anomalo ingrossamento della vena cava:

...cum interim cava ultra naturalem proportionem amplitudinis superaret aortam, quod quidem posita periodo sanguinis mirum quantam ad internas systrophas facere debuerit propensionem...

Mentre intanto la vena cava superava oltre la naturale proporzione di grandezza l'aorta, cosa che invero era strana, dato il ritmo del sangue, e quanto grande tendenza avrebbe dovuto avere in direzione del deposito interno degli umori.

(58) aeterno sur aeternas.

(59) moerentem sur + ? +

(60) provolutus sur + ?+
(61) A.S.T., Corte, Lettere Duchi e Sovrani, mazzo 66, fasc. 2. 
Egli nota come che la vena cava sia dilatata e manifesta il suo stupore per questa osservazione inattesa ricordando che il ritmo del sangue, (il flusso sanguigno) va verso il deposito interno degli umori (il cuore). Egli quindi è a conoscenza della fisiologia del circolo cavale, ma utilizza volutamente terminologie accademiche nel contesto di concezioni arcaiche.

E così molti altri sono i passaggi in cui l'osservazione corretta è subito coartata da una rigida interpretazione dottrinale ippocratico-galenica.

Bartolomeo Torrini è sicuramente un medico avvezzo alla cura dei malati e abituato all'osservazione dei decorsi clinici complessi. Se fosse lecito cancellare dal testo tutta la parte interpretativa dottrinale, avremmo un sintetico e completo decorso clinico, precisamente dispiegato nei fatti salienti di ogni giornata di malattia, puntuale nel cogliere gli elementi di variazione del quadro clinico verso il progressivo aggravamento e verso l'exitus.

Ritengo comunque che possa essere metodologicamente accettabile estrapolare dal testo torriniano i passi di stretto interesse medico, lasciando una lettura filologicamente più completa ad un altro tipo di interpretazione e valutazione. La nostra lettura infatti ha finalità medico-cliniche volte a comprendere la malattia e le cause di morte della sventurata "Colombina d'amore", e a cercare infine di stabilire se la malattia della Principessa avesse radici più lontane nel tempo a chiarimento di alcuni aspetti storiografici più generali.

La malattia ha il suo esordio qualche giorno prima del 25 di dicembre.

...Cum demum vigesima quarta decembris (Postquam per aliquot pregressas noctes in somnis calere acriter sed sine febre comperta foret) manifeste febricitavit: Iterum[que] $26^{a}$ et $28^{a}$, periodo nempe tertianaria. Vel non novit, vel sprevit ipsa dolores suos, quo tempore ob supremam valetudinem et supremum funus (heus quam lugubre) Regiae Matris supremis itidem et ipsa torquebatur angoribus. Hinc causarum cumulus ingens. Pertinaciae vigiliae, inediae moerores, atq[ue] in molli aetate mollique sexu terrores tantum non panici. Post quae tandem mensis vigesima octava perennis corripuit ignis, et febris continens, cuius proinde initium ab ea die fas est exordiri...

Quando, infine, il 24 dicembre (dopo che per alcune notti precedenti, durante il sonno era molto accaldata, ma senza che la febbre si fosse manifestata) fu colta palesemente dalla febbre e nuovamente il 26 e il 28, per l'intervallo evidentemente della terzana. Quindi o non riconobbe oppure disprezzò le proprie sofferenze, nel momento particolare, a causa della malattia incurabile riguardante la Regina Madre e del suo inevitabile (ob quanto funesto !) esito letale, ugualmente anch'essa stessa era tormentata da dolori indicibili. Da qui un accumularsi grande di concause. Costanti veglie, incapacità a trattenere il cibo, lamenti e per l'età assai giovane e la fragilità propria del suo sesso paure, per poco non panico. Dopo tali avvenimenti infine, il 28 del mese un bruciore continuo si impadroni di lei e una febbre interna dal giorno dell' inizio della quale è giusto iniziare il discorso.

Dapprima febricola, nei giorni successivi febbre accomagnata dal vomito (nel testo: incapacità a trattenere il cibo). Il 28 dicembre aumenta la febbre e il malessere. Il Torrini fa partire da questa data il conteggio delle giornate del decorso clinico. Certamente questo è corretto dal punto di vista della descrizione dell'epicrisi, ma forse riduttivo dal punto di vista anamnestico. Infatti da circa una settimana prima la giovane Duchessa presentava sintomi di febbricole notturne. Comunque le sue condizioni di salute nei 12- 15 mesi precedenti non erano perfette. La conferma ci viene dalla corrispondenza tra la duchessa Cristina e la marchesa Villa e del suo consorte, inviati a Parigi per definire il contratto di nozze. L'8 novembre 1662 essi informano che la giovane Principessa, che aveva avuto il menarca circa un anno prima con alcuni cicli regolari, presentò amenorrea causata da febbre e astenia profonda. Nel corso del 
mese di novembre le condizioni generali si erano ristabilite, ma non era cessata l'amenorrea $^{62}$. In una missiva successiva del 14 gennaio del 1663 il marchese Villa informa che la Principessa soffriva nuovamente di nausee, cefalea tanto da essere costretta al riposo. La data del matrimonio infatti fu rinviata più volte, e tra le varie cause, quella dell'indisponibilità di salute della Principessa non era ultima.

...Ad $4^{\text {am }}$ usque continens febris, et quae synocham parachimastriam simularet. $4^{a}$ diarhoea deiectiones biliosae variae. Quinta fluxus к $\alpha \tau \eta \eta v i \omega v$ intempestivus, et ganeus, febris remittitur. Sexta febris remissior, septima $\chi \alpha \tau \alpha \mu \eta v \imath \alpha$ cessant, febris intenditur deiectiones ó $\rho \tau \tau o r$, mox et $\delta v \sigma \omega ́ \delta \varepsilon \varepsilon \varsigma$, et paraceae quae humoris cacœethel r/iam testarentur.

Exacerbatis circa vesperam per totum morbi processum quotidianis accessionibus perseveratura, cum quadam sed levi circa meridiem anadyplosi. Nona lumbricus vivens, subruber, per alvum ductus, deiectiones frequentiores et variae. Decima lienteria. Undecima exacerbata omnia 'A $\lambda \iota \sigma \mu$ ó $\varsigma$ sitis inexausta, gravis et frequens anhelitus, tussis molesta, genarum rubor calor ỏ $\xi \hat{v} \varsigma$ in digitorum palpis, suspicis epigenesis in pulmones, et peripneumoniae. Interea subsultis in carpo, et motus iectigativi / $\left(f^{\circ}\right.$

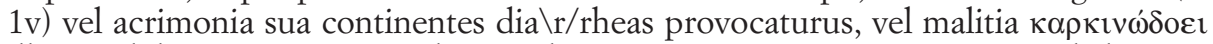
illam indolem assumpturus, de qua dictum est $\mu \eta \dot{\sigma \varepsilon \rho \alpha \pi \varepsilon ́ v \varepsilon ı v ~ \beta \varepsilon ́ \lambda \tau \imath o v, ~ v e l ~ d e n i q u e ~}$ incremento intestini aditum obstructurus cuius emphrati remoratae alvinae sordes in ileos disenterias, cruciatus, et feras anadromes pullularentur...

La febbre interna si mantenne fino al $4^{\circ}$ giorno, a simulare un versamento diffuso. Nel $4^{\circ}$ giorno diarrea ed evacuazioni di varia natura, contenenti bile. In quinta giornata un flusso mestruale nel momento non dovuto, maleodorante: la febbre cade. In sesta giornata la febbre ancora più bassa, in settima cessano iflussi mestruali, la febbre si inasprisce, feci solide, subito dopo anche umide e dolori acuti che attestano la natura maligna del liquido. Ripetutisi, aggravandosi verso la sera, per tutto il decorso della malattia gli accessi quotidiani, questo decorso andava procedendo con una qualche, ma lieve, ripetizione intorno a mezzogiorno. In nona giornata un verme vivo, di colorito rossastro, uscito attraverso l'alvo intestinale ed evacuazioni più frequenti e varie. Il decimo giorno una sorta di dissenteria. L'undicesimo giorno tutti i sintomi si aggravano: mancan$z$ a di appetito, sete irrefrenabile, pesante e frequente il respiro, tosse stizzosa, arrossamento delle guance, calore acuto nei polpastrelli delle dita, un sintomo evolutivo sospetto nei polmoni e nella zona intorno ad essi (le pleure n.d.t.). Intanto tremiti al palmo, e movimenti inquieti che con la loro forza sia tendono a provocare diarree ininterrotte, sia questo fenomeno con l'aggravamento andrà ad assumere quella natura carcinomatosa della quale si è detto la quale è detta incurabile, sia con l'accrescimento avrebbe ostruito l'adito dell'intestino con l'occlusione del quale vengono trattenute lorde feci dell'alvo nell'ileo, sofferenze feroci e numerosi soprassalti di dolore.

Fin dall'inizio considerata dal Torrini la sintomatologia che caratterizza il quadro clinico è di tipo intestinale con una componente infettivo-infiammatoria importante: febbre, vomito e diarrea. Il quadro clinico si mantiene invariato per quattro giorni. Dopo una fugace remissione di due giorni, ricompare la febbre elevata e dolori addominali assai intensi. Il quadro clinico caratterizzato dai dolori acuti a tipo colica, con variazione di intensità nel corso del tempo, rimane invariato fino alla decima giornata. Dalla undicesima il quadro muta in quello di una peritonite comparendone $\mathrm{i}$ sintomi tipici: nausea, arsura delle fauci, senso di sete irrefrenabile, febbre alta, come evidenziabile dall'arrossamento delle guance. Nel contempo i segni generali di sepsi si manifestano con respiro frequente, quale tentativo di compensare le alterazioni

(62) BB. Crohn, L. Ginzburg, G.D. OppenahiMER, Regional ileitis; a pathologic and clinical entity.
«American Journal of Medicine», 13(5): 583-90, 1952 Nov. 
metaboliche conseguenti allo stato settico, e la variazione di intensità della circolazione sanguigna, osservata laddove il circolo capillare è più facilmente visibile, nel letto ungueale e nei polpastrelli delle dita. Sono questi fenomeni vasoattivi indotti dallo stato iniziale di shock. Il quadro tossiemico progredisce con la comparsa di tremori agli arti.

Il Torrini è estremamente preciso nella descrizione sintetica dei sintomi che sono raccolti secondo la reale loro insorgenza, tanto da consentire una lettura patologica estremamente chiara. Nel passo seguente la lettura attenta, rende le osservazioni del Torrini precisissime nel descrivere l'evoluzione dei sintomi delle modificazioni metaboliche della giovane ammalata, affetta da shock settico, mentre si sta avviando progressivamente verso un quadro di acidosi metabolica che la condurrà a morte.

...Thorax angustum intra cuius parietes coarctati pulmones inspirationis remora[m] quandam experirentur, unde conclusis, nec diflatis halitibus et geminata pro angustiae compensatione antristrophe nil nisi incandescere superesset. Pulmonum substantia fusca nigredine torrefacta, arida et exsucca, livida, lurida sine sistrophe, sine tuberculo, nisi quatenus ex altero lobo expressus pauculus liquor saniei praetulit speciem, ex altero expressus cruor speciem attramenti. Verbo tota substantia tantum non sphacelata.

Haec est area in qua veluti in licto Natura haesit, et subsidiariis in viribus distituta imperio cessisse suo reapse convincitur...

Il torace oppresso all'interno delle cui pareti i polmoni costretti soffrono un certo impedimento della respirazione, da cui chiusi e non aperti i movimenti del respiro e raddoppiata per compensare la ristrettezza con l'inversione non può che generare un processo di infiammazione. Un materiale dei polmoni scuro nerastro come carbonizzato, arido e asciutto, livido, sporco, senza consistenza senza tubercolo, se non nella misura in cui da uno dei lobi un po' di liquido spremuto, presenta l'aspetto del pus, dall'altro il sangue spremuto presenta l'aspetto dell' inchiostro. Mediante una semplice parola, ogni sostanza a sè appena non mescolata.

Questo è lo spazio nel quale come su una superficie [spalmata di vischio] la Natura rimane bloccata e privata delle forze che l'aiutavano, in realtà viene costretta a rinunciare al suo dominio.

Ecco sopravvenire un'asincronia degli atti inspiratori: inspirazione più breve ed espirazione prolungata. Questo fenomeno si osserva sia come meccanismo fisiologico di compensazione dell'acidosi metabolica progressiva, sia quale fenomeno di accompagnamento della peritonite diffusa. Esso è sostenuto dalla difficoltà a muovere regolarmente i muscoli addominali e il diaframma, cooperanti nel meccanismo dell'espirazione, irrigiditi dal dolore per la reazione infiammatoria del peritoneo alla sepsi viscerale.

Insorgono forse complicanze polmonari correlate al quadro peritonitico, che tuttavia vengono forse enfatizzate dal Torrini rispetto alla loro reale importanza nel contesto del quadro clinico addominale. Di esse infatti non si trova traccia nell'esame del cadavere.

Una lucida osservazione del Torrini fà da cesura al diario dell'evoluzione drammatica della malattia. Essa è dettata certamente da una grande pratica clinica conquistata sul campo e dalla indubbia dimestichezza con la cura dei malati. Egli ha percepito che si sta passando il punto di non ritorno e afferma: "Questo è lo spazio nel quale come su una superficie spalmata di vischio, la Natura rimane bloccata e 
privata delle forze che l'aiutavano, in realtà viene costretta a rinunciare al suo dominio." A tale punto è giunta l'evoluzione della malttia che il medico comprende che la vix sanatrix Naturae, unico vero strumento terapeutico a disposizione del medico e speranza dell'ammalato, non ha più possibilità di agire.

Da questa affermazione, che è la resa incondizionata delle capacità di intervento umano alla malattia, il Torrini incomincia un lungo excursus sui meccanismi patologici che hanno determinato la gravità del quadro clinico. Questa parte delle Relazione è condotta pedestremente nell'alveo della disamina della dottrina delle discrasie degli umori atrobiliari e il loro ruolo nel determinismo della flogosi e della generazione del calore interno, causa di squilibri funzionali di tutti gli organi. Il discorso clinico riprende subito dopo con lucidità.

...Decima $4^{a}$. Paraphrenitis $\backslash 16^{a} \mathrm{coma} /$, neq[ue] tantum praestitere ex artis praescripto usurpata praesidia quae poulmonum phlogosim, humoris fervorem acrimoniam, cacoetheiam corrigerent. Phlebosomiae, blanda cathartis, refrigerantia, humectantia, renutrientia, pectoralia, cardiaca. Alexipharmaca quin post ferales tot epigeneses, $\tau \hat{\omega} v$ ỏ $\xi \dot{\varepsilon} \omega v \tau o \hat{\iota} \zeta$, tandem increscente morbo et fatiscente robore die decima octava febris prima ipsa accessionis hora fato concederet...

In quattordicesima giornata: delirio. In sedicesima coma, e non riuscirono a resistere $i$ presidi adoperati secondo la prescrizione della medicina che avrebbero dovuto combattere la flogosi dei polmoni, il ribollire dell'umore, l'acidità, la natura maligna della malattia. La cura attraverso flebotomie, un blando depurativo, sostanze rinfrescanti, idratanti, supernutrienti, emollienti per il petto, sostanze atte a stimolare il cuore. Alessifarmaci (rimedi specifici contro i veleni n.d.t.) che dopo tanti peggioramenti degli stati acuti a stati altrettanto acuti, infine aggravandosi la malattia $e$ andando a calar le forze, il diciottesimo giorno nella prima ora del peggioramento della febbre essa stessa si arrendeva al Destino.

Si giunge alla quattordicesima giornata. La paziente presenta il quadro conclamato della tossicosi settica con sofferenza cerebrale e delirio. Progressivamente il quadro evolve nei due giorni successivi (sedicesima giornata) verso il coma. Ancora un giorno in stato di incoscienza e il giorno seguente, il diciottesimo dall'esordio della malattia, la quindicenne Duchessa di Savoia Francesca d'Orleans, spirava.

Il giorno successivo, il 16 gennaio 1664, venne effettuata l'autopsia, o per meglio dire usando la terminologia del tempo, si procedette all'apertura del cadavere.

...Lubuit licuitq[ue] secto regio cadavere ea perscrutari, quae assertam pridem à Medicis

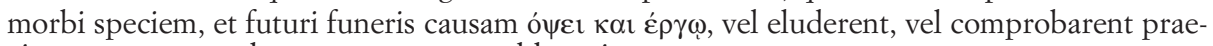
cipue vero num pulmonum aestum ac phlogosim par esset arguere...

Si ritenne giusto e lecito, dopo aver sezionato il regale cadavere di osservare, attraverso la vista e manualmente, quelle cose che negassero o comprovassero le caratteristiche della malattia, asserite prima dai medici, e la causa della imminente sepoltura, soprattutto veramente se il calore dei polmoni e la flogosi avessero uguale origine.

La prima notazione fatta dal Torrini riguarda lo stato di disidratazione delle masse muscolari come degli organi interni del cadavere, in accordo con la diagnosi di peritonite e il conseguente sequestro di liquidi circolanti negli intestini. Lo stomaco e l'intestino risultano fortemente congesti, come egli puntualmente evidenzia notandone il colorito rossastro. 
...Abdominis cuncta viscera emuncta item et arida, quaeq[ue], nec exprimenti udorem concederent. Nempe non tam vacuatis pertinaci fluxu, quam et discussis et in fulgines elevatis humoribus à fervore acerrimo, à quo et ventriculus, et intestina quandam rubedinem contraxere, veluti et hepar exanguem habitum nec non $\kappa \lambda \omega \rho \alpha ́ v$ ac pallido flavam tincturam...

Tutti gli organi interni dell' addome ugualmente ripuliti e asciutti e che non cedevano liquido neppure alle pressioni di colui che era addetto all'operazione. Veramente non tanto vuotati da un flusso inarrestabile, quanto scossi, tolti e trasformati in fuliggine questi umori dal calore fortissimo per il quale e lo stomaco e gli intestini assunsero un certo colorito rossastro, come anche il fegato assunse un aspetto esangue e assai verde, anche un colorito giallo per il pallore.

Infine emerge assai chiaramente la causa della peritonite e della morte della Principessa:

...Intestinis hoc unum non levis momenti inerat: tumor divulsus, asper carnei quantum

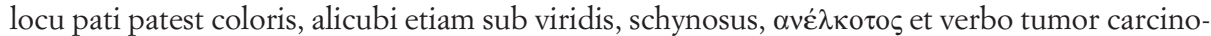
matodes, qui partem ceci et coli totum initium etiam infra valvulam intro succingebat. Soboles ista quidem tum melancholici succi, tum sordium quae in ceci bursa plus aequo grandiori collectae ab aliquot mensibus potuerunt in eius, et coli substantia systrophem parere, et ultimis hisce diebus titillamento suo alvum iugiter proritare: sed in qua tamen proclive est syderis nostri fixam perniciem serio meditare, quando hoc unum $\pi \dot{\alpha} \theta$ os preparabat id funus in paucos menses, quo fuit alterius festinatione praeoccupatu[m]...

Negli intestini questa sola cosa di non lieve importanza: il tumore lacerato, con i contorni irregolari, del colore della carne per quanto lo spazio lasciasse intravedere, altrove anche verdastro, fissurato, non ulcerato e, in una parola, tumore carcinomatoso che avvolgeva all' interno parte del cieco e tutto l'inizio del colon, anche sotto la valvola.

Questa degenerazione invero sia di un liquido di bile nera, sia di sporcizie che nella borsa del cieco più grande del normale, raccolte da alcuni mesi operarono nel suo tessuto e in quello del colon procurarono un deposito e in questi ultimi giorni col suo titillare continuo invasero l'alvo: ma bisogna meditare seriamente tuttavia da dove discende la sventura fissata della nostra stella poichè questa sola malattia preparava codesta morte in pochi mesi, in qualche modo si impadroni prima con sollecitudine di un altra.

Un tumore infiammatorio che coinvolge l'ultima ansa ileale (...anche sotto la valvola...) il cieco e parte del colon ascendente. Questo stesso tumore presenta una perforazione.

Il Torrini non parla dei segni della peritonite, come la presenza o meno di versamento o la presenza di fibrina, ma come abbiamo già notato, l'aspetto delle anse fortemente congeste è quello appunto che si osserva nelle peritoniti diffuse. Viene così aggiunto un ulteriore elemento di riscontro e di comprensione del quadro anatomopatologico della peritonite conseguente alla rottura di un viscere. Tale quadro, in considerazione di elementi epidemiologici, difficilmente può essere rappresentato da una neoplasia del colon ascendente, o del cieco, per la giovane età della paziente, mentre assai più verosimile è l'ipotesi di un tumore infiammatorio da enterocolite granulomatosa dell'intestino o morbo di Crohn. Questa ipotesi diagnostica concorda con una lunga fase di malattia prima dell'evento occlusivo terminale, caratterizzata da febbricola o febbre, dolori addominali, intensa astenia e crisi subocclusive.

Marginalmente si ricorda che la descrizione del primo caso di enterocolite granulomatosa riportato in letteratura è opera di Giovanni Battista Morgagni (1682-1771) che nella sua straordinaria opera in cinque libri "De sedibus et causis morborum per 
anatomen indagatis - Venetia, 1761" riporta la storia clinica e il referto dell'autopsia condotta su di un giovane di 20 anni con una storia clinica di diarrea protratta, talvolta sanguinolenta e dolori addominali. La causa di morte fu la perforazione dell'ileo terminale a livello della valvola ileociecale. La definizione del quadro clinico e anatomopatologico delle coliti granulomatose si ebbe ad opera di Burrill Bernard Crohn ${ }^{63}$ autore della relazione "Regional ileitis; a pathologic and clinical entity" tenuta al meeting annuale dei gastroenterologi americani a New Orleans nel 1952.

La Relazione si chiude con alcune considerazioni filosofiche poste in relazione ad enunciazioni dottrinali sulla genesi della malattia ed espressioni di compianto per i destini delle due Duchesse giunte a morte a distanza di poco più di due settimane l'una dall'altra.

Le ultime espressioni di triste commiato sono particolarmente efficaci e rendendo bene la tensione emotiva di colui che coinvolto nel dramma umano della vicenda ultima della sventurata Principessa ne prende definitivo commiato. vita fatiscat...

...Ergo et Calamus corruat ac aeterno moeronum \in tumulo/ provolutus cum principe

Quindi anche la penna cada e rotolata nell'eterna tomba eretta da coloro che piangono, dissolva la vita con la principessa.

Si noti che Calamus nel manoscritto ha l'iniziale maiuscola quale personalizzazione stessa del Torrini. 